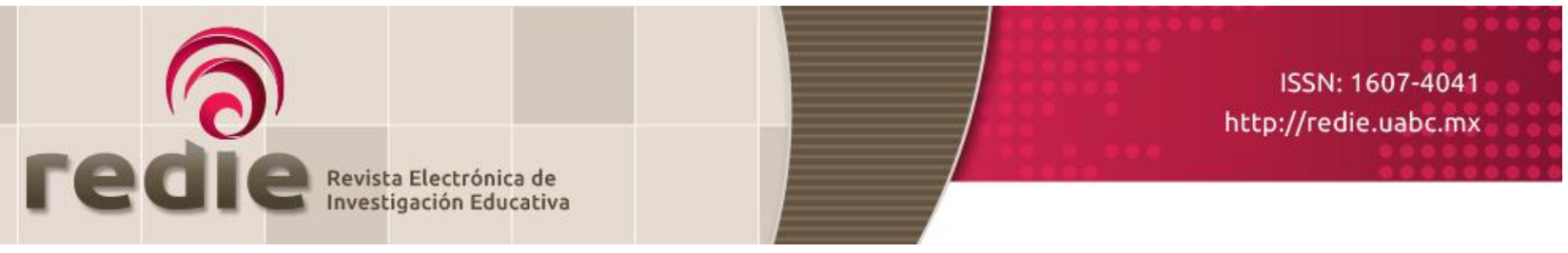

Vol. 19, Núm. 2, 2017

\title{
Validación de un instrumento para evaluar la autopercepción del pensamiento crítico en estudiantes de Medicina
}

\author{
Validation of an Instrument to Assess Self-Perception \\ of Critical Thinking in Students of Medicine
}

Silvia Lizet Olivares Olivares (*) solivares@itesm.mx

Mildred Vanessa López Cabrera (*) mildredlopez@itesm.mx

(*) Tecnológico de Monterrey

(Recibido: 6 de agosto de 2014; Aceptado para su publicación: 30 de noviembre de 2015)

Cómo citar: Olivares, S. L. y López, M. V. (2017). Validación de un instrumento para evaluar la autopercepción del pensamiento crítico en estudiantes de Medicina. Revista Electrónica de Investigación Educativa, 19(2), 67-77.

https://doi.org/10.24320/redie.2017.19.2.848

\begin{abstract}
Resumen
El acelerado ritmo de generación de conocimiento requiere que los estudiantes de Medicina desarrollen competencias genéricas durante su formación. El pensamiento crítico es primordial para establecer juicios autorregulados basándose en argumentación fundamentada. Para medir la disposición de estudiantes en competencias, se desarrolló el Cuestionario de Competencias Genéricas Individuales (ccal), el cual mide con 74 reactivos la autopercepción de los estudiantes hacia las competencias de: pensamiento crítico, alfabetización informacional, autodirección, administración del tiempo, solución de problemas y toma de decisiones. Рara validar la sección de pensamiento crítico 18 expertos evaluaron el cuestionario y determinaron la pertinencia de cada aseveración; además, se aplicó el instrumento a 135 estudiantes. El Alfa de Cronbach fue 0.739 y el análisis factorial resultó en 3 componentes asociados a dimensiones del pensamiento crítico: Interpretación y análisis de información, Juicio de una situación con datos objetivos y subjetivos, e Inferencia de consecuencias de la decisión basándose en el juicio autorregulado. La estadística descriptiva indica que los estudiantes se perciben mejor en sus preferencias para evaluar e inferir.
\end{abstract}

Palabras clave: Pensamiento crítico, educación superior, educación médica.

\section{Abstract}

The fast pace at which knowledge is generated means that students of medicine must develop generic skills during the course of their training. Critical thinking is essential to make self-regulated judgments based on substantiated reasoning. In order to measure students' skills, the Individual Generic Skill Questionnaire (CCGI, in Spanish) was developed, with 74 items that measure students' self-perception in the following skills: critical thinking, information literacy, self-management, time management, problem solving and decision-making. To validate the critical thinking section, 18 experts assessed the questionnaire and determined the relevance of each assertion, and the questionnaire was given to 135 
students. Cronbach's alpha was 0.739 and a factorial analysis produced 3 components associated with different facets of critical thinking: information interpretation and analysis, making a judgment on a situation from objective and subjective information, and inferring the consequences of a decision based on self-regulated judgments. Descriptive statistics show that student self-perception is better regarding their preferences in assessing and inferring.

Keywords: Critical thinking, higher education, medical education.

\section{Introducción}

El pensamiento crítico es altamente deseable en los estudiantes de Medicina. De acuerdo con Facione y Facione (2008) el pensamiento clínico es el resultado del uso adecuado del pensamiento crítico tanto en las habilidades cognitivas como en la disposición hacia el mismo, y Gupta y Upshur (2012) coinciden en la importancia del pensamiento crítico en los profesionales de la salud para la toma de decisiones profesionales y educativas.

Para definir el pensamiento crítico, Facione (1990) parte del consenso de la American Psychological Association (APA), "la formación de un juicio autorregulado para un propósito específico, cuyo resultado en términos de interpretación, análisis, evaluación e inferencia pueden explicarse según la evidencia, conceptos, métodos, criterios y contexto que se tomaron en consideración para establecerlo" (p. 2). De acuerdo con la APA, las seis dimensiones del pensamiento crítico son: interpretación, análisis, evaluación, inferencia, explicación y autorregulación. En este consenso se complementan las habilidades del pensamiento con las características que disponen a un individuo para convertirse en un pensador crítico.

Рara Paul y Elder (2006), "el pensamiento crítico es un conjunto de habilidades intelectuales, aptitudes y disposiciones (...) [que lleva] al dominio del contenido y el aprendizaje profundo" (p. 9). Esta propuesta considera al pensamiento crítico como una serie de estándares para que los individuos aprendan en forma autodirigida y con motivación a lo largo de su vida con humildad, autonomía, integridad, coraje, perseverancia, confianza, empatía y justicia. Además de estas actitudes intelectuales, los autores enlistan cinco habilidades que debe tener el pensador crítico tales como: 1) plantear preguntas y problemas esenciales, 2) recopilar y evaluar la información relevante, 3) llegar a conclusiones razonadas, 4) pensar de manera abierta reconociendo y evaluando las implicaciones, y 5) comunicar efectivamente las alternativas de solución a problemas complejos.

De acuerdo con Norris y Ennis (1989), el pensamiento crítico se refiere al proceso de discriminar cuál es la "verdad" que cada individuo juzga conveniente creer. Los autores desglosan el pensamiento crítico en tres partes fundamentales: a) un resultado que puede ser interpretado como la decisión acerca de la creencia o la acción, b) una fundamentación de la decisión y c) la conexión lógica entre la decisión y los argumentos para tomarla, y enumeran una serie de disposiciones hacia el pensamiento crítico, tales como: uso de fuentes de información, búsqueda de alternativas, búsqueda de razones, consideración de la situación total, orden en la complejidad de las partes, enfoque a puntos relevantes y originales, razonamiento sobre puntos en los que tiene desacuerdo, sensibilidad a los sentimientos de otros y apertura para considerar otros puntos de vista aunque no coincidan con los propios.

Para Garrison, Anderson y Archer (2001) el pensamiento crítico es un proceso y un resultado, y establecen cuatro etapas de aplicación práctica para desarrollarlo: 1) evento de activación a través de un dilema o problema, 2) exploración de información, 3) integración de la conclusión y 4) solución y comunicación para iniciar un nuevo ciclo.

En el caso de la formación de estudiantes de Medicina, el pensamiento crítico requiere la inclusión de ambas perspectivas: la de la disposición del pensamiento crítico y la de las habilidades del pensamiento (Krupat et al., 2011). 
A partir de una integración de las definiciones anteriores podemos decir que el pensamiento crítico es la elaboración de un juicio sustentado en datos objetivos y subjetivos previamente interpretados y analizados, lo cual facilita al individuo inferir las consecuencias de sus propias decisiones.

De acuerdo con la clasificación de la tabla I, el proceso de interpretación en el contexto del pensamiento crítico requiere de humildad intelectual para iniciar un proceso de búsqueda de información en diversas fuentes, así como perseverancia y persistencia para encontrar los resultados esperados (Facione, 1990). En la etapa de análisis, el pensador crítico requiere confianza y motivación para estructurar cada una de las partes de información considerando diversos enfoques. Para la evaluación e inferencia es importante que el pensador crítico cuente con flexibilidad y apertura para considerar todos los puntos de vista en forma honesta y justa, aunque sean divergentes con su postura. Para ello, además necesita tener la capacidad de cambiar de opinión cuando sea prudente según la evidencia. Por último, la explicación requiere de cierta decisión y coraje intelectual para enfrentar al grupo con argumentos sólidos. Este proceso siempre puede ser mejorado a través de la autorregulación, la cual requiere de autonomía y autoconfianza para aceptar cada una de las responsabilidades adquiridas.

Tabla I. Atributos del pensador crítico de acuerdo con los elementos teóricos

\begin{tabular}{|c|c|c|c|}
\hline $\begin{array}{l}\text { Elementos del } \\
\text { pensamiento crítico } \\
\text { (Facione, 1990) }\end{array}$ & $\begin{array}{l}\text { Características del } \\
\text { pensador crítico } \\
\text { (Norris y Ennis, 1989) }\end{array}$ & $\begin{array}{l}\text { Disposición al } \\
\text { pensamiento crítico } \\
\text { (Facione, 2011) }\end{array}$ & $\begin{array}{l}\text { Disposición al } \\
\text { pensamiento crítico } \\
\text { (Paul y Elder, 2006) }\end{array}$ \\
\hline $\begin{array}{l}\text { 1) Interpretación: } \\
\text { búsqueda en diversas } \\
\text { fuentes de información }\end{array}$ & $\begin{array}{l}\text { Usa fuentes de información } \\
\text { y las cita; trata de estar bien } \\
\text { informado }\end{array}$ & $\begin{array}{l}\text { Búsqueda continua de } \\
\text { información; honestidad } \\
\text { para establecer los propios } \\
\text { prejuicios y tendencias } \\
\text { egoístas }\end{array}$ & $\begin{array}{l}\text { Humildad intelectual: } \\
\text { distingue lo que sabe de } \\
\text { lo que no sabe }\end{array}$ \\
\hline $\begin{array}{l}\text { 1) Interpretación: } \\
\text { perseverancia en el } \\
\text { manejo de alternativas }\end{array}$ & Busca alternativas & $\begin{array}{l}\text { Curiosidad ante una amplia } \\
\text { gama de cuestiones }\end{array}$ & $\begin{array}{l}\text { Perseverancia intelectual: } \\
\text { aprenden a trabajar a } \\
\text { pesar de las frustraciones }\end{array}$ \\
\hline $\begin{array}{l}\text { 2) Análisis: confianza } \\
\text { y estructura en la } \\
\text { investigación }\end{array}$ & $\begin{array}{l}\text { Busca razones; considera la } \\
\text { situación total; } \\
\text { ordena la complejidad de las } \\
\text { partes; no pierde de vista el } \\
\text { enfoque de los puntos } \\
\text { relevantes y originales }\end{array}$ & $\begin{array}{l}\text { Confianza y motivación } \\
\text { hacia los procesos de } \\
\text { investigación }\end{array}$ & $\begin{array}{l}\text { Confianza en la razón: } \\
\text { uso de las facultades } \\
\text { racionales }\end{array}$ \\
\hline $\begin{array}{l}\text { 3) Evaluación: } \\
\text { flexibilidad y apertura }\end{array}$ & $\begin{array}{l}\text { Razona sobre puntos en los } \\
\text { que tiene desacuerdo; es } \\
\text { sensible a los sentimientos }\end{array}$ & $\begin{array}{l}\text { Flexibilidad para } \\
\text { considerar alternativas } \\
\text { y opiniones }\end{array}$ & $\begin{array}{l}\text { Empatía: da entrada } \\
\text { empática a los puntos } \\
\text { de vista que difieren de } \\
\text { los propios }\end{array}$ \\
\hline 3) Evaluación: justicia & No aplica & $\begin{array}{l}\text { Justicia para evaluar } \\
\text { el razonamiento }\end{array}$ & Justicia de pensamiento \\
\hline $\begin{array}{l}\text { 4) Inferencia: considera } \\
\text { todas las opciones }\end{array}$ & $\begin{array}{l}\text { Tiene apertura para: } \\
\text { considerar otros puntos de } \\
\text { vista aunque esté en } \\
\text { desacuerdo con los mismos }\end{array}$ & $\begin{array}{l}\text { Mentalidad abierta sobre } \\
\text { visiones divergentes }\end{array}$ & $\begin{array}{l}\text { Integridad intelectual: se } \\
\text { sujeta a los mismos } \\
\text { estándares que el resto }\end{array}$ \\
\hline $\begin{array}{l}\text { 4) Inferencia: puede } \\
\text { cambiar de opinión }\end{array}$ & $\begin{array}{l}\text { Toma una posición y la } \\
\text { cambia cuando la evidencia } \\
\text { es suficiente }\end{array}$ & $\begin{array}{l}\text { Es prudente para } \\
\text { suspender, hacer o alterar } \\
\text { sus propios juicios }\end{array}$ & No aplica \\
\hline $\begin{array}{l}\text { 5) Explicación: decisión } \\
\text { para establecer postura }\end{array}$ & No aplica & No aplica & $\begin{array}{l}\text { Coraje intelectual: } \\
\text { dispuesto a desafiar la } \\
\text { desaprobación del grupo }\end{array}$ \\
\hline $\begin{array}{l}\text { 6) Autorregulación: } \\
\text { dueño de su realidad }\end{array}$ & No aplica & $\begin{array}{l}\text { Autoconfianza } \\
\text { en sus capacidades } \\
\text { de razonamiento }\end{array}$ & $\begin{array}{l}\text { Autonomía intelectual: } \\
\text { es responsable de su } \\
\text { forma de pensar, creencias } \\
\text { y valores }\end{array}$ \\
\hline
\end{tabular}

Рara los profesores clínicos el pensamiento crítico en los estudiantes implica habilidades tales como: obtención de información de diferentes fuentes, integración y síntesis de la información considerando diferentes opciones de tratamiento, comunicación con pacientes, toma de decisiones basadas en la 
evidencia y actuación identificando sus propios prejuicios. Según Krupat et al. (2011) algunas de las actitudes que un pensador crítico no debe tener son: insensibilidad a la complejidad, toma de decisiones con información incompleta, confianza en una sola perspectiva, falta de humildad para cambiar de opinión y actuar a la defensiva, entre otros.

En la educación médica, aunque es un consenso la necesidad de formación del pensamiento crítico, diversos autores coinciden en las dificultades para medirlo en forma integral (Garrison, Anderson y Archer, 2001; Gupta y Upshur, 2012). Algunas de las herramientas más utilizadas para medir la disposición al pensamiento crítico son el Watson-Glaser Critical Thinking Appraisal (WGCTA) y el Critical Thinking Disposition Inventory (CCTDI). El WGCTA tiene una confiabilidad en un rango de 0.66 a 0.87 y cinco subescalas: inferencia, suposiciones, deducción, interpretación y evaluación de argumentos (Burris, 2005). Por su parte, el CCTDI contiene 75 ítems con siete escalas: indagación, apertura, sistematicidad, análisis, búsqueda de la verdad, autoconfianza para el pensamiento crítico y madurez. Los niveles de Alfa de Cronbach reportados varían entre 0.60 y 0.78 en cada subescala y 0.90 en la prueba general (Facione, Facione y Sánchez, 1994). Estas pruebas para entornos no necesariamente de educación médica, además de ser costosas requieren un tiempo considerable de administración debido a su longitud y miden una competencia única, por ello, en la escuela de Medicina bajo estudio, se diseñó el Cuestionario de Competencias Genéricas Individuales (ccal) el cual mide con 74 reactivos la autopercepción de los estudiantes hacia las competencias de: pensamiento crítico, alfabetización informacional, autodirección, administración del tiempo, solución de problemas y toma de decisiones. La intención de la institución es contar con mecanismos para identificar la predisposición de los estudiantes a otras competencias en forma transversal, además de las conceptuales propias de la disciplina.

\section{Método}

El objetivo de la investigación fue diseñar y validar ítems de la sección de pensamiento crítico del ccal para medir la autopercepción de los estudiantes de Medicina de una universidad privada sobre el nivel de la disposición a esta competencia y presentar un análisis descriptivo de los resultados. El diseño del estudio es de tipo cuantitativo, transeccional, descriptivo y no experimental. Como participantes se seleccionó a la población de alumnos del sexto año de la carrera de Médico Cirujano de la universidad bajo estudio, 135 en total. Se siguió la metodología aplicada por Fisher, King y Tague (2001) en las siguientes fases: 1) diseño de un modelo de disposición a la competencia, 2) determinación de los ítems para evaluar el desempeño de los estudiantes de acuerdo al modelo propuesto, 3) validación por expertos y mejora de los ítems, y 4) aplicación de la prueba piloto y análisis de estadístico.

\subsection{Diseño de un modelo de pensamiento crítico}

Una persona no carece de una competencia de forma absoluta, sino que la domina en cierto grado (Wagennar y González, 2003), por lo que en este estudio nos proponemos evaluar la autopercepción de los estudiantes como pensadores críticos en tres dimensiones: 1) interpretación y análisis de información, 2) juicio de una situación con datos objetivos y subjetivos, y 3) inferencia de consecuencias de la decisión basándose en el juicio autorregulado, las cuales se sustentan en las propuestas de diversos autores (Facione y Facione, 2008; Norris y Ennis, 1989; Gupta y Upshur, 2012; Garrison et al., 2001).

En cuanto a la dimensión Interpretación y análisis de la información, la interpretación se refiere a una forma básica de aprendizaje que involucra entendimiento de experiencias, situaciones, información, creencias y eventos (Facione, 2011). En lugar de memorizar un concepto, requiere entender su significado y expresarlo de manera simplificada o modelarlo para ser capaces de entenderlo en un contexto mayor. Según Facione (2011), el análisis requiere identificar la relación entre los argumentos, preguntas, conceptos y descripciones que han sido ya cubiertos de un concepto en particular. Esta habilidad involucra la determinación de un marco de referencia para comparar argumentos o versiones; entre más variables haya para comparar, mayor cantidad de análisis será requerida, por lo que aumenta la posibilidad de identificar similitudes o diferencias entre ellos.

En cuanto a la dimensión Juicio de una situación específica con datos objetivos y subjetivos, se refiere a un 
proceso donde se tiene la capacidad de juzgar la relevancia de cada argumento u opinión analizada (Facione, 2011). Por lo que se requiere evaluar las propuestas usando estrategias deductivas o inductivas. El pensamiento deductivo usa la certeza de los hechos y el juicio autorregulado de la información presentada de un análisis previo, mientras que el inductivo evalúa un resultado aunque se carezca de información, usando mecanismos subjetivos como el sentido común o las creencias propias; es preferible que el acercamiento presente un balance.

La dimensión Inferencia de las consecuencias de la decisión basándose en el juicio autorregulado propicia una conclusión razonable tomando en consideración información objetiva y subjetiva, creencias, opiniones y argumentos (Facione, 2011). Involucra el establecimiento de un nuevo nivel de conocimiento que construya alternativas, decisiones o planes de acción. Se recomienda un autoanálisis o reflexión de los procesos metodológicos y cognitivos para desarrollar la mejora continua. La figura 1 representa el modelo para evaluar el pensamiento crítico.

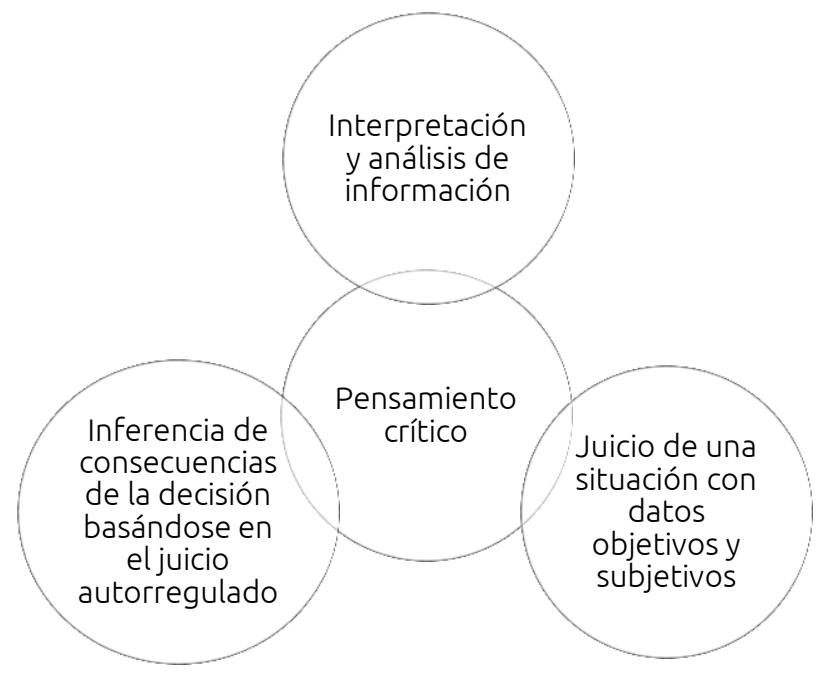

Figura 1. Modelo de tres dimensiones para evaluar el pensamiento crítico en estudiantes de Medicina

\subsection{Determinación de los ítems para evaluar el desempeño de los estudiantes de acuerdo al modelo propuesto}

En esta fase se desarrolló el instrumento de 74 reactivos para medir la autopercepción del estudiante sobre su disposición a las competencias: alfabetización informacional, solución de problemas, administración del tiempo, autodirección, toma de decisiones y pensamiento crítico. Se seleccionó la escala de Likert de cinco respuestas, que van de "totalmente de acuerdo" a "totalmente en desacuerdo". Algunas de las preguntas tenían sentido positivo y otras negativo. El instrumento se aplicó en el mes de enero de 2013. En el estudio se presentan sólo los resultados de la sección que pretende medir la disposición al pensamiento crítico, la cual fue desarrollada con un total de 13 aseveraciones de acuerdo a la teoría previamente presentada. Estos reactivos estaban orientados a medir las actitudes y autopercepción de los estudiantes ante la interpretación y análisis de la información (reactivos 1 al 4), sus preferencias para evaluar e inferir (reactivos 5 al 9) y las posturas frente a las habilidades de explicación y autorregulación (reactivos 10 al 13) (Facione, 1990). Las aseveraciones relacionadas con los campos clínicos (reactivos 4, 6 y 8) están soportados por las investigaciones de Krupat et al. (2011) y Gupta y Upshur (2012). En la tabla II se presenta de forma sintetizada la teoría de la que surge cada dimensión del modelo, así como los respectivos ítems. 
Tabla II. Surgimiento de los reactivos con base en la teoría analizada

\begin{tabular}{ll}
\hline Componente & Facione (1990) \\
\hline Interpretación & La habilidad de interpretación \\
y análisis de & evalúa que el estudiante \\
información & comprenda y exprese el \\
& significado de experiencias, \\
& juicios y creencias, y de ahí \\
& obtener un significado que \\
& pueda ser categorizado o \\
& descrito. \\
& La habilidad de análisis incluye la \\
& revisión de ideas, identificación \\
& de argumentos y análisis de \\
& dichos argumentos.
\end{tabular}

Paul y Elder (2006)

El estándar 1: propósitos, metas y

objetivos, obtiene como resultado que los alumnos expresen en su propio lenguaje el propósito de lo que sucede en el aula, la materia, un problema de estudio y los problemas de su vida diaria. Además de ser capaces de direccionar sus acciones para alcanzar nuevas metas.

El estándar 2: preguntas, problemas y asuntos, contempla que los estudiantes expresen con sus propias palabras la pregunta de investigación, tengan claridad y precisión en la expresión del problema analizado, distingan las preguntas conceptuales de las factuales, así como los contenidos de acuerdo al nivel de relevancia.

En el estándar 3: información, datos, evidencia y experiencia, los estudiantes deben expresar la información más importante de forma resumida. De igual forma, pueden distinguir entre hechos, inferencias, datos, investigación y evidencia.

Juicio de una
situación
específica con
datos objetivos
y subjetivos

La habilidad de la evaluación consiste en la valoración de argumentos y posturas. La habilidad de autorregulación incluye que el estudiante sea capaz de hacer un autoanálisis e identifique áreas con oportunidad de mejora en su propio desempeño. principios, definiciones, leyes y axiomas, los

\section{Reactivos propuestos}

1. Entro en pánico cuando tengo que lidiar con algo muy complejo. 2. Puedo explicar con mis propias palabras lo que acabo de leer. 3. Prefiero el detalle a la síntesis.

4. Puedo hacer comparación entre diferentes métodos o tratamientos.

5. Utilizo mi sentido común para juzgar la relevancia de la información.

\section{El estándar 5: suposiciones y}

presuposiciones, los estudiantes identifican suposiciones, ya sean propias o de terceros, En el estándar 6: conceptos, teorías, estudiantes identifican un concepto, son capaces de ejemplificarlo y desarrollarlo. En el estándar 8:puntos de vista y marcos de referencia, los estudiantes definen su punto de vista, reconocen la posibilidad de que existan diferentes puntos de vista, y a esa luz analizan críticamente el propio.
6. Prefiero la medicina basada en evidencia a mi percepción personal.

7. Si hay cuatro razones a favor y la mía es en contra, apoyo las cuatro.

8. Puedo determinar un diagnóstico aunque no tenga toda la información. 9. A pesar de los argumentos en contra, mantengo firmes mis creencias.

10. Evado ser crítico para evitar conflictos. 12. Prefiero aplicar un método conocido antes de arriesgarme a probar uno nuevo.
En el estándar 4: inferencias e

interpretaciones, los estudiantes son capaces de distinguir entre inferencias y conclusiones, así como distinguir entre la consistencia o grado de confusión que representan.

En el estándar 7: implicaciones y consecuencias, los alumnos identifican las implicaciones y consecuencias de su desempeño, sean estas positivas o negativas, probables o improbables. Siendo precedido de un análisis razonado sobre dichas implicaciones.

\section{Expreso}

alternativas innovadoras a pesar de las reacciones que pueda generar. 13. Sé distinguir entre hechos reales y prejuicios. requiere la recopilación de
evidencia, el armar conjeturas como diferentes alternativas de solución. obtenidos, así como sostener un proceso de argumentación. explicar y justificar resultados de la decisión basándose en autorregulado 


\subsection{Validación de ítems, prueba piloto y análisis estadístico}

Se aplicó la primera fase del Método de Delphi, solicitando la revisión de los ítems a un grupo de 18 expertos -profesores de la carrera de Educación superior, experiencia en diseño curricular y que aceptaran participar voluntariamente en el estudio-, quienes revisaron el contenido en cuanto a relevancia, representatividad y claridad. Se les solicitó que indicaran en una escala Likert de cinco puntos el grado en el que consideraban si las declaraciones eran adecuadas para medir la competencia de pensamiento crítico, siendo 1 totalmente de acuerdo y 5 totalmente en desacuerdo. Esta información fue codificada y analizada utilizando MinitabR 17. El criterio utilizado fue considerar como válidos los reactivos en los que al menos el $80 \%$ de los expertos seleccionaban valores de 3 o menos en la escala de Likert.

El instrumento se aplicó en enero del 2013 a la población total del sexto año de la carrera de Médico Cirujano de una universidad privada. Tres métodos fueron seleccionados para el análisis estadístico: Alfa de Cronbach, análisis de factores y estadística descriptiva.

Para determinar la consistencia interna tanto de la sección de pensamiento crítico como del cçı se utilizó el coeficiente Alfa de Cronbach, en el cual un valor superior a 0.70 es satisfactorio para la mayoría de los casos (Vogt, 2007). Se calculó también la correlación ítem-total para evaluar si cada ítem en la sección particular era consistente con los demás de la misma sección. Entre más altos los valores del coeficiente, más claro es que ese ítem pertenece a esa escala.

Posteriormente, se hizo un análisis factorial para revisar las categorías a las que se asociaban los reactivos, aplicando el criterio de Morales (2013). El análisis de aplicado fue el de componentes principales. Los factores extraídos fueron determinados usando gráfico de sedimentación. Se aplicó una rotación Varimax para obtener una estructura más simple. Aunque el autor afirma que con N>100 0.3 sería el valor mínimo admitido, se usó un valor de 0.4 para obtener una estructura más limpia.

Por último, se utilizó la estadística descriptiva para analizar los resultados de las respuestas de los estudiantes. Considerando las respuestas con valores más cercanos a 1 como respuestas favorables, encontrándose más cercanas a "totalmente de acuerdo". Las preguntas escritas en sentido negativo fueron recodificadas para hacer estas comparaciones.

\section{Resultados}

De acuerdo con los resultados de la evaluación de la sección de pensamiento crítico realizada por el grupo de 18 expertos, se conservaron 10 de las 13 aseveraciones originales. Además, 8 ítems se reclasificaron a otra dimensión de la competencia. La comparación entre el diseño original del instrumento y el que resultó después de elaborar el estudio y análisis estadístico se muestra a detalle en la tabla III. 
Tabla III. Comparación del diseño original de la herramienta contra los resultados obtenidos

\begin{tabular}{|c|c|c|}
\hline ítem & $\begin{array}{l}\text { Dimensión de competencia a la que } \\
\text { pertenecía el ítem cuando se } \\
\text { diseñó el instrumento }\end{array}$ & $\begin{array}{l}\text { Dimensión de competencia a la que } \\
\text { pertenece el ítem después del } \\
\text { análisis del instrumento }\end{array}$ \\
\hline $\begin{array}{l}\text { 1. Entro en pánico cuando tengo } \\
\text { que lidiar con algo muy complejo. }\end{array}$ & $\begin{array}{l}\text { 1) Interpretación y análisis de } \\
\text { información. }\end{array}$ & $\begin{array}{l}\text { 1) Interpretación y análisis de } \\
\text { información }\end{array}$ \\
\hline $\begin{array}{l}\text { 2. Puedo explicar con mis propias } \\
\text { palabras lo que acabo de leer. }\end{array}$ & $\begin{array}{l}\text { 1) Interpretación y análisis de } \\
\text { información. }\end{array}$ & $\begin{array}{l}\text { 2) Juicio de una situación específica } \\
\text { con datos objetivos y subjetivos }\end{array}$ \\
\hline 3. Prefiero el detalle a la síntesis. & $\begin{array}{l}\text { 1) Interpretación y análisis de } \\
\text { información. }\end{array}$ & Eliminado \\
\hline $\begin{array}{l}\text { 4. Puedo hacer comparación entre } \\
\text { diferentes métodos o tratamientos. }\end{array}$ & $\begin{array}{l}\text { 1) Interpretación y análisis de } \\
\text { información. }\end{array}$ & $\begin{array}{l}\text { 2) Juicio de una situación específica } \\
\text { con datos objetivos y subjetivos }\end{array}$ \\
\hline $\begin{array}{l}\text { 5. Utilizo mi sentido común рагa } \\
\text { juzgar la relevancia de la } \\
\text { información. }\end{array}$ & $\begin{array}{l}\text { 1) Interpretación y análisis de } \\
\text { información. }\end{array}$ & $\begin{array}{l}\text { 2) Juicio de una situación específica } \\
\text { con datos objetivos y subjetivos }\end{array}$ \\
\hline $\begin{array}{l}\text { 6. Prefiero la Medicina basada en } \\
\text { evidencia a mi percepción personal. }\end{array}$ & $\begin{array}{l}\text { 2) Juicio de una situación específica } \\
\text { con datos objetivos y subjetivos. }\end{array}$ & $\begin{array}{l}\text { 2) Juicio de una situación específica } \\
\text { con datos objetivos y subjetivos }\end{array}$ \\
\hline $\begin{array}{l}\text { 7. Si hay cuatro razones a favor y la } \\
\text { mía es en contra, apoyo las cuatro. }\end{array}$ & $\begin{array}{l}\text { 2) Juicio de una situación específica } \\
\text { con datos objetivos y subjetivos. }\end{array}$ & liminado \\
\hline $\begin{array}{l}\text { 8. Puedo determinar un diagnóstico } \\
\text { aunque no tenga toda la } \\
\text { información. }\end{array}$ & $\begin{array}{l}\text { 2) Juicio de una situación específica } \\
\text { con datos objetivos y subjetivos. }\end{array}$ & $\begin{array}{l}\text { 3) Inferencia de las consecuencias de } \\
\text { la decisión basándose en el juicio } \\
\text { autorregulado }\end{array}$ \\
\hline $\begin{array}{l}\text { 9. A pesar de los argumentos en } \\
\text { contra, mantengo firmes mis } \\
\text { creencias. }\end{array}$ & $\begin{array}{l}\text { 2) Juicio de una situación específica } \\
\text { con datos objetivos y subjetivos. }\end{array}$ & $\begin{array}{l}\text { 3) Inferencia de las consecuencias de } \\
\text { la decisión basándose en el juicio } \\
\text { autorregulado }\end{array}$ \\
\hline $\begin{array}{l}\text { 10. Evado ser crítico para evitar } \\
\text { conflictos }\end{array}$ & $\begin{array}{l}\text { 2) Juicio de una situación específica } \\
\text { con datos objetivos y subjetivos. }\end{array}$ & Eliminado \\
\hline $\begin{array}{l}\text { 11. Expreso alternativas innovadoras } \\
\text { a pesar de las reacciones que pueda } \\
\text { generar. }\end{array}$ & $\begin{array}{l}\text { 3) Inferencia de las consecuencias de } \\
\text { la decisión basándose en el juicio } \\
\text { autorregulado. }\end{array}$ & $\begin{array}{l}\text { 2) Juicio de una situación específica } \\
\text { con datos objetivos y subjetivos }\end{array}$ \\
\hline $\begin{array}{l}\text { 12. Prefiero aplicar un método } \\
\text { conocido antes de arriesgarme a } \\
\text { probar uno nuevo. }\end{array}$ & $\begin{array}{l}\text { 2) Juicio de una situación específica } \\
\text { con datos objetivos y subjetivos. }\end{array}$ & $\begin{array}{l}\text { 1) Interpretación y análisis de } \\
\text { información }\end{array}$ \\
\hline $\begin{array}{l}\text { 13. Sé distinguir entre hechos reales } \\
\text { y prejuicios. }\end{array}$ & $\begin{array}{l}\text { 3) Inferencia de las consecuencias de } \\
\text { la decisión basándose en el juicio } \\
\text { autorregulado. }\end{array}$ & $\begin{array}{l}\text { 2) Juicio de una situación específica } \\
\text { con datos objetivos y subjetivos }\end{array}$ \\
\hline
\end{tabular}

Los reactivos eliminados fueron: 3. Prefiero el detalle a la síntesis, perteneciente a la dimensión de Interpretación y análisis de información; 7. Si hay cuatro razones y la mía es en contra, apoyo las cuatro; y 10. Evado ser crítico para evitar conflictos, perteneciente a la dimensión de Juicio de una situación específica con datos objetivos y subjetivos. Los cuales fueron eliminados en la validación de los expertos; sin embargo, guarda una correspondencia con las respuestas ofrecidas por los alumnos, ya que son los reactivos con mayor dispersión, con una desviación estándar de 1.23, 1.20 y 1.18, respectivamente.

Se midió la confiabilidad de la sección de los 10 reactivos resultantes mediante el Alfa de Cronbach, el cual resultó en 0.739 para la sección de pensamiento crítico. La confiabilidad total del ccGı es de 0.790, lo que indica que la prueba de la sección analizada es confiable tanto en lo general como en lo particular.

El análisis de componentes principales resultó en tres factores (ver tabla IV). El componente asociado a Interpretación y análisis de información agrupó los reactivos 1 y 9, los cuales evalúan negativamente el orden para trabajar con asuntos complejos y la flexibilidad para considerar otras alternativas (Facione, 1990; Krupat et al., 2011). La componente "Juicio de una situación con datos objetivos y subjetivos" se asocia a los reactivos 2, 3, 4, 5 y 10, los cuales se relacionan en forma positiva con la autoconfianza de las propias habilidades para razonar (Facione, 1990). Por último, se agruparon los reactivos 6 y 7, que valoran en forma negativa la capacidad reflexividad sobre las creencias básicas propias, en la componente "Inferencia de consecuencias de la decisión basándose en el juicio autorregulado" (Norris y Ennis, 1989; Krupat et al., 2011). 
Tabla IV. Análisis factorial

\begin{tabular}{|c|c|c|c|}
\hline ítem & $\begin{array}{l}\text { Interpretación } \\
\text { y análisis de } \\
\text { información }\end{array}$ & $\begin{array}{l}\text { Juicio de una } \\
\text { situación específica } \\
\text { con datos objetivos } \\
\text { y subjetivos }\end{array}$ & $\begin{array}{l}\text { Inferencia de las } \\
\text { consecuencias de la } \\
\text { decisión con base en el } \\
\text { juicio autorregulado }\end{array}$ \\
\hline $\begin{array}{l}\text { 1. Entro en pánico cuando tengo que } \\
\text { lidiar con algo muy complejo. }\end{array}$ & 0.646 & -0.352 & 0.046 \\
\hline $\begin{array}{l}\text { 2. Puedo explicar con mis propias palabras } \\
\text { lo que acabo de leer. }\end{array}$ & 0.194 & 0.678 & 0.307 \\
\hline $\begin{array}{l}\text { 3. Puedo hacer comparación entre } \\
\text { diferentes métodos o tratamientos. }\end{array}$ & 0.094 & 0.750 & 0.017 \\
\hline $\begin{array}{l}\text { 4. Utilizo mi sentido común para juzgar la } \\
\text { relevancia de la información. }\end{array}$ & 0.309 & 0.649 & -0.056 \\
\hline $\begin{array}{l}\text { 5. Prefiero la Medicina basada en evidencia } \\
\text { a mi percepción personal. }\end{array}$ & 0.242 & 0.434 & -0.642 \\
\hline $\begin{array}{l}\text { 6. Puedo determinar un diagnóstico aunque } \\
\text { no tenga toda la información. }\end{array}$ & -0.453 & 0.309 & 0.473 \\
\hline $\begin{array}{l}\text { 7. A pesar de los argumentos en contra, } \\
\text { mantengo firmes mis creencias. }\end{array}$ & 0.350 & 0.302 & 0.500 \\
\hline $\begin{array}{l}\text { 8. Expreso alternativas innovadoras a pesar } \\
\text { de las reacciones que pueda generar. }\end{array}$ & -0.282 & 0.620 & 0.024 \\
\hline $\begin{array}{l}\text { 9. Prefiero aplicar un método conocido antes de } \\
\text { arriesgarme a probar uno nuevo. }\end{array}$ & 0.466 & 0.002 & 0.177 \\
\hline $\begin{array}{l}\text { 10. Sé distinguir entre hechos reales y } \\
\text { prejuicios. }\end{array}$ & -0.126 & 0.568 & -0.356 \\
\hline
\end{tabular}

El análisis descriptivo incluye los resultados de cada reactivo. Considerando que se midió a toda la población, los resultados miden la percepción de la misma sobre predisposiciones al pensamiento crítico. La tabla $V$ muestra los resultados de la media y la desviación estándar de cada reactivo. Asimismo, se calcula el Alfa de Cronbach eliminando cada ítem para evaluar si el instrumento se vería mejorado al descartar dicho reactivo. 
Tabla V. Resultados de estadística descriptiva para cada uno de los ítems

\begin{tabular}{|c|c|c|c|c|}
\hline & Ítem & Promedio & $\begin{array}{l}\text { Desviación } \\
\text { estándar }\end{array}$ & $\begin{array}{l}\text { Alfa de } \\
\text { Cronbach } \\
\text { si se elimina } \\
\text { el ítem }\end{array}$ \\
\hline \multirow{3}{*}{$\begin{array}{l}\text { Dimensión asociada } \\
\text { a Interpretación y } \\
\text { análisis de } \\
\text { información }\end{array}$} & \multirow{3}{*}{$\begin{array}{l}\text { 1. Entro en pánico cuando tengo que lidiar } \\
\text { con algo muy complejo. } \\
\text { 9. Prefiero aplicar un método conocido antes } \\
\text { de arriesgarme a probar uno nuevo. } \\
\text { Total por nivel }\end{array}$} & 3.89 & 1.187 & .796 \\
\hline & & 2.52 & 1.084 & .790 \\
\hline & & 3.21 & & \\
\hline \multirow{7}{*}{$\begin{array}{l}\text { Dimensión asociada } \\
\text { al Juicio de una } \\
\text { situación específica } \\
\text { con datos objetivos } \\
\text { y subjetivos }\end{array}$} & $\begin{array}{l}\text { 2. Puedo explicar con mis propias palabras } \\
\text { lo que acabo de leer. }\end{array}$ & 1.54 & .897 & .784 \\
\hline & $\begin{array}{l}\text { 3. Puedo hacer comparación entre diferentes } \\
\text { métodos o tratamientos. }\end{array}$ & 1.47 & .683 & .782 \\
\hline & $\begin{array}{l}\text { 4. Utilizo mi sentido común para juzgar la } \\
\text { relevancia de la información. }\end{array}$ & 1.38 & .728 & .783 \\
\hline & $\begin{array}{l}\text { 5. Prefiero la Medicina basada en evidencia } \\
\text { a mi percepción personal. }\end{array}$ & 1.53 & .797 & .785 \\
\hline & $\begin{array}{l}\text { 8. Expreso alternativas innovadoras a pesar } \\
\text { de las reacciones que pueda generar. }\end{array}$ & 2.01 & .973 & .783 \\
\hline & $\begin{array}{l}\text { 10. Sé distinguir entre hechos reales y } \\
\text { prejuicios. }\end{array}$ & 1.60 & .883 & .785 \\
\hline & Total por nivel & 1.58 & & \\
\hline \multirow{3}{*}{$\begin{array}{l}\text { Dimensión asociada } \\
\text { al Inferencia de las } \\
\text { consecuencias de la } \\
\text { decisión basándose } \\
\text { en el juicio } \\
\text { autorregulado }\end{array}$} & $\begin{array}{l}\text { 6. Puedo determinar un diagnóstico aunque } \\
\text { no tenga toda la información. }\end{array}$ & 2.62 & 1.004 & .787 \\
\hline & $\begin{array}{l}\text { 7. A pesar de los argumentos en contra, } \\
\text { mantengo firmes mis creencias. }\end{array}$ & 2.34 & 1.127 & .787 \\
\hline & Total por nivel & 2.48 & & \\
\hline
\end{tabular}

\section{Discusión}

La confiabilidad de la sección de pensamiento crítico del ccal fue de 0.739, el cual es equiparable con las pruebas internacionales WGCTA que presenta un rango de 0.66 a 0.87 y con la California Critical Thinking Disposition Inventory (CCTDI) con rango entre 0.60 y 0.78 en cada subescala. Una vez validadas el resto de las secciones del cuestionario ccal, este será de utilidad para medir en forma longitudinal durante su estancia en el programa la disposición de los alumnos hacia cada una de las seis competencias que mide. Es indispensable acompañar la educación basada en competencias con elementos para su evaluación y seguimiento para asegurar el aprendizaje a lo largo de la vida de los estudiantes. La principal aportación de este instrumento es que se logró medir tres dimensiones del pensamiento crítico con tan solo 10 reactivos.

La información obtenida corresponde a la primera valoración por parte de expertos y la primera aplicación para estudiantes, lo cual puede considerarse como una primera prueba piloto en la cual se observó alta coincidencia entre los reactivos que fueron eliminados por los expertos y los reactivos con alta varianza; es decir, los resultados están limitados a esta primera aproximación.

Los resultados de estadística descriptiva muestran que los ítems con respuestas más favorables (más cercanos a totalmente de acuerdo) son el 3, 4 y 5, que corresponden al componente "Juicio de una situación con datos objetivos y subjetivos". Esto quiere decir que los alumnos se perciben mejor en sus habilidades para analizar y juzgar la relevancia de argumentos.

A futuro, se planea ampliar la muestra para corroborar si los factores que tuvieron niveles menores a 0.3 en el análisis factorial pueden permanecer en el cuestionario. Los resultados de la estadística descriptiva, muestran que los alumnos se basan principalmente en el sentido común para revisar la relevancia de la información, aunque establecen que utilizan el proceso de comparación como estrategia de análisis de la 
información. Si bien es importante utilizar el sentido común para evaluar la información (en el momento de determinar un diagnóstico médico) es importante basarse en la evidencia y la información. En la siguiente versión del cuestionario serán reforzados los cuestionamientos relacionados con la flexibilidad para aceptar los puntos de vista de otros y el uso de los datos para el establecimiento de evaluaciones y juicios.

\section{Referencias}

Burris, S. (2005). Effect on problem based learning on critical thinking ability and content knowledge acquisition of secondary agriculture students (Tesis doctoral, Universidad de Missouri-Columbia.

Facione N. C., Facione P. A. y Sánchez C. A. (1994) Critical thinking disposition as a measure of competent clinical judgment: the development of the California Critical Thinking Disposition Inventory. The Journal of Nursing Education, 33(8), 345-350.

Facione, N. C., y Facione, P. A. (2008). Critical thinking and clinical reasoning in the health sciences: a teaching anthology. CA: California Press.

Facione, P. A. (1990). Critical thinking: a statement of expert consensus for purposes of educational assessment and instruction. CA: The California Academic Press.

Facione, P. A. (2011). Critical thinking: What it is and why it counts. Recuperado de http://www.insightassessment.com

Fisher, M., King, J. y Tague, G. (2001). Development of a self-directed learning readiness scale for nursing education. Nurse Education Today, 21, 516-525.

Garrison, D. R., Anderson, T. y Archer, W. (2001) Critical thinking, cognitive presence, and computer conferencing in distance education. The American Journal of Distance Education, 15(1), 7-23.

Gupta, M. y Upshur, R. (2012). Critical thinking in clinical medicine: what is it? Journal Of Evaluation In Clinical Practice, 18(5), 938-944. doi:10.1111/j.1365-2753.2012.01897.

Krupat, E., Sprague, J. M., Wolpaw, D., Haidet, P., Hatem, D., y O’Brien, B. (2011). Thinking critically about critical thinking: ability, disposition or both? Medical Education, 45, 625-635.

Morales, P. (2013). El análisis factorial en la construcción e interpretación de tests, escalas y cuestionarios. Madrid: Universidad Pontificia Comillas.

Norris, S. y Ennis, R. H. (1989). Evaluating critical thinking. Pacific Grove, CA: Midwest Publications.

Paul, R. y Elder, L. (2006). Critical thinking competency standards. Dillon Beach, CA: The Foundation of Critical Thinking.

Vogt, P. (2007). Quantitative research methods for professionals. Boston, MA: Pearson/Allyn and Bacon.

Wagennar, R. y González, J. (2003). Tuning educational structures in Europe. España: Universidad de Deusto. 